\title{
Autolesão na adolescência e a produção científica nacional: revisão integrativa da literatura*
}

\author{
Loraine Seixas Ferreira** \\ Gislaine Chaves*** \\ Leila Salomão de La Plata Cury Tardivo***
}

\begin{abstract}
Resumo
A autolesão é um comportamento com início geralmente na adolescência e tem como característica principal lesões provocadas no próprio corpo com o intuito de aliviar as angústias, reduzir emoções negativas e, até mesmo, como uma forma do adolescente comunicar seu sofrimento, quando este não pode ser expresso de outra forma. Embora possa gerar ferimentos graves, muitos autores compreendem o comportamento autolesivo como "não suicida" por não ter a intensão consciente de morte. $\mathrm{O}$ interesse pelo tema da autolesão na adolescência tem aumentado nos últimos anos e, dessa forma, o objetivo desse trabalho foi realizar uma revisão integrativa de artigos produzidos sobre esse tema, provenientes de pesquisas nacionais, no período de 2015 a 2020 nas bases de dados BVS, SCIELO, PEPSIC e Portal de Periódicos CAPES. Foram encontrados apenas 12 trabalhos, os quais compreenderam a autolesão como um fenômeno multifatorial e realizada como uma forma de aliviar angústias que não podem ser verbalizadas.
\end{abstract}

Palavras-chave: autolesão, conduta autolesiva, adolescência

\section{Self-injury in adolescence and national scientific production: literature integrative revision}

\begin{abstract}
Self-injury is a behaviour that often appears at the beginning of adolescence and has as main characteristic injuries caused in the body itself in order to relief the anguish, reduce negative emotions and even as a way for adolescents to communicate their suffering when it cannot be expressed in another way. Although it can generate severe injuries, many authors understand self-injurious behavior as "non suicidal" because they do not have the conscious intention of death. The concern around adolescence self-injury has increased in recent years and, therefore, the aim of this work was to carry out an integrative review from articles produced about on this topic, from national research, in the period from 2015 and 2020 in the BVS, SCIELO, PEPSIC and CAPES Journal Portal data bases. Only 12 works were found, which understood self-injury as a multifactorial phenomenon performed as a way of relieving anxieties that cannot be verbalized.
\end{abstract}

Keywords: Self-Injury, Self-Injurious Behavior, adolescent

* Trabalho realizado com apoio da Coordenação de Aperfeiçoamento de Pessoal de Nível Superior - Brasil (CAPES) - Código de Financiamento 001 ** ORCID iD https://orcid.org/0000-0003-2580-5721. Instituto de Psicologia da Universidade de São Paulo - IPUSP. Doutoranda do Departamento de Psicologia Clínica (Instituto de Psicologia da Universidade de São Paulo, São Paulo, Brasil). Bolsista CAPES. E-mail: loraine.seixas@gmail.com.

*** ORCID iD http://orcid.org/0000-0002-9239-8401. Instituto de Psicologia da Universidade de São Paulo - IPUSP. Doutoranda do Departamento de Psicologia Clínica (Instituto de Psicologia da Universidade de São Paulo, São Paulo, Brasil). Bolsista CAPES. Email: gislaine.ch@gmail.com .

**** ORCID iD https://orcid.org/0000-0002-8391-0610. Instituto de Psicologia da Universidade de São Paulo - IPUSP. Professora Associada do departamento de Psicologia Clínica (Instituto de Psicologia da Universidade de São Paulo, São Paulo, Brasil). E-mail: tardivo@usp.br . 


\section{Introdução}

Para estudar a adolescência é necessário defini-la em suas perspectivas psicológica, biológica e social, integrada ao processo evolutivo do ser humano, no qual a criança caminha pouco a pouco para a vida adulta, aliando o ambiente e sua história vivida (Levinsky, 1998, Tardivo, 2007). Conforme o adolescente vai perdendo sua condição de criança, sua identidade infantil, vai definindo a identidade adulta e, durante a busca dessa nova identidade, as imagens parentais introjetadas, proporcionadas pelo mundo externo satisfatório, permitirão ao adolescente elaborar as crises internas pelas quais irá vivenciar (Aberastury, 1983; Erikson, 1968/1976; Tardivo, 2007). Essas crises levam a conflitos que poderão ser mais ou menos intensos, resultado da qualidade do desenvolvimento, da estabilidade dos afetos, somado às gratificações, frustrações e da adaptação gradativa às exigências ambientais (por exemplo familiar e escolar), desde os primeiros anos de vida (Aberastury, 1983, Tardivo, 2007).

Os conflitos vivenciados na adolescência, associados a uma sociedade com uma estrutura social que pode ser mais fragilizada, produz cada vez mais comportamentos de riscos no público adolescente, por exemplo os comportamentos autolesivo, que tem seu início frequentemente já no começo da adolescência (American Psychiatric Association, 2014; Brown \& Plener, 2017; Giusti, 2013). Ao desenvolver mecanismos mais eficientes, que ajudam o adolescente a enfrentar as situações negativas, esse comportamento pode cessar, mas, em outros casos, o comportamento autolesivo pode persistir por anos e, nos casos mais graves, pode ocasionar a morte do adolescente (Giusti, 2013).

Apesar de algumas pesquisas apontarem um aumento desse comportamento, o que o que leva muitos autores a considerarem a autolesão uma epidemia (Brown \& Plener, 2017; Hawton, Saunders, \& O’Connor, 2012), o comportamento autolesivo ainda é pouco estudado no Brasil e, ainda hoje, não há um levantamento dos índices de autolesão em adolescentes no país. O Ministério da Saúde publica periodicamente dados sobre violência auto infligida, na qual pode-se incluir a autolesão. Os dados do Ministério da Saúde $(2013,2017)$ demonstram um aumento desse tipo de violência: entre os anos 2009 a 2011, nas cidades pesquisadas (24 capitais, Distrito Federal e 11 municípios), a lesão autoprovocada foi notificada em $9 \%$ de todos os atendimentos dos indivíduos entre 10 e 19 anos, sendo 9,6\% entre as meninas e 7,7\% entre os meninos (Ministério da Saúde, 2013). Os dados mais recentes são da pesquisa de 2013 a 2014 e demonstram que a violência autoprovocada foi notificada em 12,5\% dos atendimentos (10,3\% no sexo masculino e 13,7\% no sexo feminino) (Ministério da Saúde, 2017). Ou seja, os dados comprovam o aumento de violência auto infligida no público adolescente.

As caraterísticas desse comportamento, segundo a $5^{a}$ edição do Diagnóstico de Saúde Mental (American Psychiatric Association, 2014), são as lesões provocadas no próprio corpo que, geralmente, têm como objetivo reduzir emoções negativas (tensão, ansiedade, autocensura), relacionando-se também com dificuldade interpessoal ou autopunição, acompanhada de uma sensação imediata de alívio. Esse comportamento leva o adolescente a se machucar intencionalmente, podendo gerar a ferimentos graves, no entanto, sem a intenção consciente de suicídio (Arcoverde \& Soares, 2012). Nesse sentido, o DSM-5 classifica o comportamento como "autolesão não suicida", explicando que o indivíduo provoca os ferimentos sem intenção de morte declarada (American Psychiatric Association, 2014). O comportamento autolesivo é diferenciado do suicídio, como apresentado por Kovács (2008), que descreve a lesão autoprovocada como uma tentativa de restaurar o significado da angústia e, assim, uma "tentativa de sobreviver" e evitar a morte.

Estudos apontam alguns fatores relacionados ao comportamento autolesivo, sendo eles: eventos negativos ao longo da vida e conflitos familiares (abandono, violências, relação familiar disfuncional, desvalorização do adolescente pela família) e fatores sociais (bullying, influência dos pares com o mesmo comportamento, dificuldades de socializar) (Hawton et al, 2012; Tardivo et al, 2019a). Viver em ambiente inseguros, inconsistentes e marcado por negligência e violências influenciam negativamente o desenvolvimento do adolescente, podendo levá-lo a apresentar pouca habilidade para regular as emoções, manifestando assim comportamentos mal adaptados, por exemplo o comportamento autolesivo (Giusti, 2013; Hawton et al, 2012; Tardivo et al, 2019a).

As características pessoais do adolescente também são relacionadas pelos autores com o comportamento autolesivo, por exemplo a insegurança, baixa autoestima, instabilidade emocional, sentimento de culpa, negação dos pensamentos angustiantes, incapacidade para lidar com as emoções, dificuldade em sustentar um sentimento de bem-estar e de se sentir pertencente, além de fatores 
psiquiátricos, como sintomas de ansiedade e de depressão (Giusti, 2013; Guerreiro \& Sampaio, 2013; Hawton et al, 2012; Tardivo et al, 2019a). Nesse sentido, segundo Guerreiro e Sampaio (2013), a autolesão está associada ao triplo fracasso: no campo individual, familiar e social, que resulta em uma tentativa distorcida de mudar essa situação insustentável.

Os episódios de autolesão geralmente são precedidos de um aumento da ansiedade, depressão, raiva de si e sensação de perda de controle, que acarreta a realização dos cortes. Após os episódios, é comum os adolescentes relatarem sensação de bem-estar e alívio momentâneo do sofrimento, deslocamento do foco da dor emocional para a dor física, mas também sentimento de culpa, vergonha e tristeza por ter se cortado (Giusti, 2013; Kaplan, Sadock, \& Grebb 1997). Os estudos de Tardivo et al (2019a) acrescentam que os adolescentes estudados apresentaram maior dificuldade para controlar seus impulsos, no contato com a realidade e com o sofrimento, buscando o controle das emoções de forma destrutiva. Dessa forma, esses adolescentes encontram na dor e nos ferimentos o restabelecimento de um sentido, além de buscar nos cortes um contorno para o corpo recuperando o limite entre o dentro e o fora (Bernal, 2019; Le Breton, 2010).

Esse comportamento também pode ser compreendido como um pedido de ajuda do adolescente, que demonstra nos cortes uma pista de suas aflições, que não podem ser postas em palavras, restando o apelo de uma comunicação primitiva. Dessa forma, o outro, quem irá receber a "mensagem", tem grande importância. No entanto, muitas vezes esse outro é ausente, faltando um espaço de ressonância no qual o adolescente possa ser compreendido e, ao não encontrar palavras e nem a quem comunicar seu sofrimento, resta-lhe mostrar ao invés de dizer (Bernal, 2019; Le Breton, 2010; Tardivo et al, 2019a). Sendo assim, um espaço de escuta é fundamental para adolescentes que apresentem o comportamento autolesivo, convocando o olhar do outro para o fenômeno (Bernal, 2019).

Diante ao exposto, compreende-se que a adolescência é uma etapa de grande importância no desenvolvimento, que deve ser integrada pelas perspectivas psicológicas, biológicas e sociais, na qual a subjetividade do adolescente, o ambiente no qual está inserido e sua história são crucias para entender as dificuldades dessa fase. A autolesão ocorre em meio aos conflitos vivenciados pelos adolescentes, associados à experiência de viver em uma sociedade com estruturas enfraquecidas (Tardivo et al., 2019a).

Apesar de o interesse pelo tema da autolesão ter aumentado nos últimos anos, o que possibilitou novas pesquisas publicadas e em andamento, no Brasil ainda há carência de estudos que contribuam para a maior compreensão do comportamento autolesivo e que apresentem a prevalência desse comportamento na população adolescente e pré-adolescente. Dessa forma, esse trabalho tem como o objetivo realizar uma revisão integrativa de artigos produzidos acerca da autolesão em adolescentes, publicados em português, portanto de fácil acesso à população brasileira, provenientes de pesquisas nacionais e tem sua relevância por apurar dados que permitam refletir sobre o que se tem estudado sobre o comportamento autolesivo no Brasil nos últimos anos e como os pesquisadores compreendem o fenômeno.

\section{Método}

O trabalho se baseia em uma revisão integrativa de literatura cientifica nacional referente aos estudos do comportamento autolesivo em adolescentes, tendo como fator de destaque a reflexão e a integração da produção científica, de forma que ajude na produção de pesquisas futuras. Para sua realização, foram utilizados os seguintes Descritores em Ciências da Saúde (DeCS): autolesão OR comportamento autolesivo OR comportamento autodestrutivo AND adolescência OR adolescente.

Foram pesquisados trabalhos no idioma português (por ser mais fácil o acesso pela população brasileira em geral), com limitador temporal no período de publicação de janeiro de 2015 até dezembro de 2020, consultados nas bases de dados: Biblioteca Virtual da Saúde (BVS) (utiliza as bases de dados LILACS, MEDLINE, entre outras), SCIELO, PEPSIC e Portal de Periódicos CAPES (inclui as bases de dados PEP, Web of Science, entre outras).

A revisão dos estudos seguiu as seis etapas propostas por Mendes, Silveira e Galvão (2008): (1) identificação do problema e determinação da hipótese a ser estudada, que nesse trabalhou foi se houve aumento da produção de pesquisas brasileiras sobre autolesão na adolescência, nos últimos anos, e como os pesquisadores compreendem esse comportamento. (2) estabelecimento dos critérios de inclusão e exclusão de literaturas a serem consideradas no estudo, (3) categorização dos estudos, definindo as informações que serão retiradas; (4) avaliação das litera- 
turas que foram incluídas no estudo; (5) interpretação dos resultados encontrados e (6) síntese do conhecimento.

Os trabalhos encontrados nas bases de dados pesquisadas foram considerados aptos para a pesquisa ao abordarem o tema pesquisado (autolesão em adolescentes), dentro do período de tempo estipulado, publicados em português e oriundos de trabalhos nacionais. Dessa forma, os trabalhos de outros países, mesmo que publicados em periódicos brasileiros e em língua portuguesa, entraram nos critérios de exclusão, pois essa pesquisa visa a produção nacional acerca da autolesão. Também forma excluídos trabalhos que apresentaram a autolesão como característica associada a algum transtorno específico (por exemplo, Transtorno de Personalidade Borderline, Transtorno do Espectro Autista, Transtorno no Movimento Esteriotipado).

A categorização do presente estudo se iniciou com a procura dos artigos por meio dos descritores escolhidos, realizada entre os dias 20 e 27 de dezembro de 2020. Foram encontrados 60 artigos: BVS (24 artigos), SCIELO (12 artigos), PEPSIC (07 artigos) e Portal de Periódicos CAPES (17 artigos). Após a aplicação dos critérios de inclusão e retirando as duplicidades, foram excluídos 23 artigos. A partir de então, foi iniciada a etapa de leitura dos 37 trabalhos mantidos. Em um primeiro momento foi realizada a leitura minuciosa do resumo dos artigos, visando selecionar apenas aqueles que abordassem o tema central da pesquisa. Os trabalhos que atenderam esse objetivo foram lidos na íntegra e, os trabalhos que apresentaram os dados pertinentes a esse estudo foram categorizados para posterior interpretação e sínteses. Ao final do procedimento, foram selecionados 12 artigos. A apresentação dos dados obtidos durante a seleção dos artigos está esquematizada na Figura 1 (anexo).

Após a leitura na íntegra, seis artigos foram excluídos por não atenderam ao tema pesquisado (autolesão em adolescentes): dois trabalhos visaram a ideação suicida e suicídio em adolescentes, um tinha como foco principal os riscos dos desafios da internet, outro apresenta a atenção ao adolescente que apresenta comportamentos de acting out, um artigo apresentava os desafios do COVID na saúde mental de adolescentes privados de liberdade e, por fim, o último trabalho excluído tinha como ponto central a ciência da psicanálise.

\section{Resultados e Discussão}

Tendo categorizado os estudos e selecionado aqueles que se adequaram aos objetivos do trabalho, pode-se notar que nenhum trabalho sobre o tema foi publicado no ano de 2015. Em 2016 dois artigos foram encontrados, em 2017 apenas um, 2018 três trabalhos, em 2019 também foram três publicações e, em 2020, até a data do levantamento, três estudo havia sido publicado. Apesar da pouca quantidade de trabalhos encontrados, o que demonstra a falta de pesquisas sobre o tema no Brasil, nota-se que há um aumento nas produções sobre a autolesão na adolescência, demonstrando um maior interesse pelo tema nos três últimos anos. A tabela 1 (anexo) apresenta a análise qualitativa dos artigos incluídos para esta pesquisa, contendo: autores e ano de publicação, objetivo do estudo, metodologia aplicada e principais resultados.

Avaliando os artigos trabalhados, os dados demonstram que das 12 publicações encontradas, dez tiveram como objetivo compreender os fatores relacionados à autolesão, sejam eles com foco aos fatores extrínsecos ao indivíduo (por exemplo, como os blogs podem incentivar os adolescentes, como estudo de Otto \& Santos, 2016), mas a maioria considera o fenômeno como multifatorial (como nos estudos de Cardoso, Demantova, \& Maia, 2016; Fonseca, Silva, Araújo, \& Botti, 2018; Lopes \& Teixeira, 2019; Moraes et al., 2020; Moreira, Vale, Caixeta, \& Teixeira, 2020; Santos \& Faro, 2018; Silva \& Botti, 2017; Tardivo, Rosa, Ferreira, Chaves, \& Pinto Júnior, 2019b; Tostes, Assis, Aiello Vaisberg, \& Corbett, 2018). Três artigos tiveram como objetivo compreender a autolesão dentro do ambiente escolar: um visou trabalhar como a autolesão é manifestada na escola (Lopes \& Teixeira, 2019), outro se preocupou em pesquisar o trabalho do psicólogo escolar com os adolescentes que apresentam esse comportamento (Sant'Ana, 2019) e o terceiro teve como finalidade identificar a percepção de professores e profissionais da saúde sobre o comportamento de autolesão (Gabriel, Costa, Campeiz, Salim, Silva, \& Carlos, 2020).

Quanto ao tipo de estudo realizado, cinco foram trabalhos teóricos (Cardoso et al, 2016; Moreira, et al. 2020; Santos \& Faro, 2018; Sant'Ana, 2019; Silva \& Botti, 2017) e sete realizaram pesquisa empírica (Fonseca et al, 2018; Gabriel et al, 2020; Lopes \& Teixeira, 2019; Moraes et al., 2020; Otto \& Santos, 2016; Tardivo et al, 2019b; Tostes et al, 2018). Os trabalhos teóricos se 
distribuíram de maneira uniforme ao longo do tempo pesquisado (um em cada ano, entre 2016 e 2020); já os trabalhos empíricos, foram os estudos mais frequentes a partir de 2018 (dois trabalhos em cada ano, entre 2018 e 2020). Dos trabalhos empíricos apenas um foi quantitativo (Fonseca et al, 2018), enquanto os outros seis foram pesquisas qualitativas. Desses últimos, duas publicações focaram seus estudos nos conteúdos encontrados em blogs e escritos por adolescentes que se autolesionam (Otto \& Santos, 2016; Tostes et al, 2018), outros dois tiveram como foco o ambiente escolar - um com rodas de conversas com profissionais da educação e também da saúde (Gabriel et al, 2020), e o outro realizou entrevistas com uma adolescente que apresentava o comportamento autolesivo (Lopes \& Teixeira, 2019) - um dos trabalhos qualitativos utilizou instrumentos projetivos e escalas de depressão e ansiedade com os próprios adolescentes com o comportamento (Tardivo et al, 2019b) e, por fim, um estudo buscou compreender, com os próprios adolescentes, o sentido e fatores de risco para o comportamento autolesivo (Moraes et al., 2020).

Acerca dos trabalhos teóricos, um não descreveu como foi realizado o estudo (Cardoso et al, 2016) e os outros quatro foram revisões de literatura: dois trabalharam com revisão integrativa (Moreira, et al. 2020; Silva \& Botti, 2017) e dois com revisão narrativa (Santos \& Faro, 2018; Sant'Ana, 2019) dos conteúdos encontrados em bases de dados. Desses quatro, um utilizou publicações que tinham com foco a atuação do psicólogo escolar (Sant'Ana, 2019), dois pesquisaram os fatores de risco no comportamento autolesivo (Moreira, et al. 2020; Silva \& Botti, 2017) e o último não apresentou um foco específico nos descritores usados (Santos \& Faro, 2018).

Além da adolescência, abordada em todos os trabalhos, por ser esse um critério de inclusão para esta pesquisa, um trabalhou também apresentou a autolesão em crianças (Santos \& Faro, 2018), um em adultos (Santos \& Faro, 2018) e dois em idosos (Santos \& Faro, 2018; Silva $\&$ Botti, 2017). No entanto, como apresentado por Giusti (2013), Brown e Plener (2017) e também pelo DSM-5 (American Psychiatric Association, 2014), os trabalhos pesquisados também consideram que a autolesão é mais frequente em adolescentes (Santos \& Faro, 2018; Silva \& Botti, 2017) principalmente no público feminino (Fonseca et al, 2018, Moraes et al., 2020). No entanto, Moreira et al. (2000) ressalta a falta de estudos sobre o tema da autolesão em adolescentes do sexo masculino.
Conforme apresentado na tabela, os resultados dos artigos compreenderam a autolesão como um ato não suicida, embora seja um fator de risco para o suicídio devido sua gravidade (Fonseca et al, 2018; Silva \& Botti, 2017). Apesar de alguns adolescentes realizarem ferimentos graves, a intenção de morte não é declarada (American Psychiatric Association, 2014; Arcoverde \& Soares, 2012), pois o adolescente que apresenta o comportamento autolesivo, no momento que realiza os cortes, não quer dar fim a sua vida e sim tentar sobreviver, restaurando o significado da angústia (Kovács, 2008).

Assim como discutido por Le Breton (2010), Tardivo et al (2019a) e Bernal (2019), em alguns trabalhos os cortes foram compreendidos como uma forma de comunicar ou sinalizar o sofrimento que sentem, como um pedido de ajuda, proteção e uma tentativa de terem suas angústias compreendidas (Santos \& Faro, 2018; Tardivo et al, 2019b). Já a dor causada pelos cortes foi apresentada pelos estudos como uma forma do adolescente aliviar seus sentimentos e/ou sensações negativas, como por exemplo o sentimento de vazio, sendo os cortes um recurso defensivo frente a esse sofrimento (Demantova, \& Maia, 2016; Fonseca et al, 2018; Lopes \& Teixeira, 2019; Moreira et al, 2020; Santos \& Faro, 2018; Tardivo et al, 2019b). Esse recurso demonstra a dificuldade do adolescente em controlar suas emoções, o que é feito de forma destrutiva, causando um alívio momentâneo dos sentimentos, mas que depois do alívio surgem os sentimentos de culpa, vergonha e tristeza por não ter conseguido controlar os impulsos autodestrutivos (Giusti, 2013; Kaplan \& Sadock, 1998; Tardivo et al, 2019a). O estudo de Cardoso et al (2016) ainda apresentou a dor como uma forma do adolescente se apropriar de seu corpo, buscando um contorno que recuperar o limite entre eu e não eu, como descrito por Le Breton (2010) e Bernal (2019).

Quanto aos fatores que podem estar relacionados ao início dos autocortes, a maioria dos autores que estudaram esse aspecto apontam que o comportamento autolesivo pode estar ligado a vivência de um ambiente hostil, marcado por faltas e privações, tanto no âmbito familiar quando no social (Santos \& Faro, 2018; Moraes et al., 2020; e Moreira et al., 2020; Tardivo et al, 2019b; Tostes et al, 2018). Os autores apontam que esses ambientes podem estar relacionados aos graves conflitos familiares, ausência de um dos pais, conflitos com a figura materna, falta de suporte familiar ou aos adolescentes sentirem que 
não recebem o carinho e afeto por parte do outro e uso de álcool e drogas a família (Gabriel et al, 2020; Lopes \& Teixeira, 2019; Moraes et al., 2020; e Moreira et al., 2020; Tardivo et al, 2019b; Tostes et al, 2018). A situação se agrava quando os adolescentes estão expostos a algum tipo de violência, dentre elas a negligência familiar e o bullying (Moraes et al., 2020; e Moreira et al., 2020; Silva \& Botti, 2017; Tardivo et al, 2019b). Hawton et al (2012), Giusti (2013) e Tardivo et al (2019a) já haviam demonstrado o quanto o ambiente inadequado (com conflitos, violência, inseguro, inconsistentes) são prejudiciais ao adolescente, que buscam na autolesão uma forma de dar conta de suas aflições.

Assim como apresentado anteriormente (Giusti, 2013; Guerreiro \& Sampaio, 2013; Hawton et al, 2012, Tardivo et al, 2019a), os trabalhos utilizados no levantamento bibliográfico também compreenderam a autolesão como associada a sintomas de depressão e ansiedade (Silva \& Botti, 2017, Tardivo et al, 2019b; Moraes et al., 2020; e Moreira et al., 2020). Algumas características individuais também foram apontadas pelos autores como frequente em adolescentes que praticam autolesão: baixa autoestima, dificuldade de se expressarem verbalmente, impulsividade isolamento social e sentimento de solidão (Moraes et al., 2020; e Moreira et al., 2020; Tardivo et al, 2019b).

Um outro fator associado à autolesão apontado pelos autores foi a influência dos pares (Gabriel et al, 2020; Moraes et al., 2020; e Moreira et al., 2020; Santos \& Faro, 2018), sendo a internet, representada por blogs e redes sociais, apontada como um fator de grande relevância, por ser esse um espaço de trocas e para que os adolescentes possam se expressar livremente, sem se sentirem julgamentos (Gabriel et al, 2020; Moraes et al, 2020; Otto \& Santos, 2016, Tostes et al, 2018,). Diante o exposto, assim como apresentado por Guerreiro e Sampaio (2013) e Santos \& Faro (2018), o comportamento autolesivo deve ser considerado como multicausal por estar relacionado a vários setores vivenciados pelo adolescente: familiar, social e individual.

Por fim, sobre o tratamento, Moreira et al (2000) destaca a carência de estudos sobre estratégias de prevenção e terapêutica. No entanto, a escuta das angústias desses adolescentes foi compreendida como forma de favorecer o movimento criativos desses jovens (Gabriel et al, 2020; Lopes \& Teixeira, 2019), assim como incluir os familiares e educadores no tratamento desses adolescentes (Sant'Ana, 2019). Retomando Bernal (2019) é fundamental que esses adolescentes se sintam vistos e acolhidos em seus sofrimentos, não necessitando dos cortes para comunicar suas angústias.

\section{Considerações Finais}

Apesar das poucas produções encontradas, foi possível compreender a autolesão como multifatorial, ou seja, relacionada a todos os âmbitos da vida do adolescente: familiar, social e pessoal. Sendo assim, é relevante observar o quanto a vivência em ambiente (familiar e social) hostil, no qual o adolescente está exposto às violências de diferentes tipos, é um fator preocupante para a saúde mental do adolescente, assim como os transtornos de depressão e ansiedade.

Também foi possível compreender a autolesão como uma forma de aliviar angústias que não podem ser verbalizadas, seja por não conseguir dar voz ao sofrimento, ou por não ter a quem comunicar o sofrimento. Nesse sentido, os cortes também aparecem como uma forma de comunicar as aflições e, sendo assim, o espaço de escuta para esses adolescentes é imprescindível, para que possam externalizar sua dor de forma mais adaptativa.

O estudo com os trabalhos publicados no período de 2015 a 2020 permitiu observar que, embora o interesse pelo tema da autolesão esteja aumentando nos últimos anos, ainda há poucas produções sobre o assunto, o que também pode ser prejudicada pela falta de consenso na forma de designar o comportamento, dificultando a busca por trabalhos sobre a autolesão em adolescentes. Além de pesquisas teóricas sobre o comportamento autolesivo, também é necessário que sejam realizados mais trabalhos empíricos, visando a compreensão da autolesão na perspectiva do próprio adolescente, dando voz aos jovens que apresentam esse comportamento.

\section{Referências}

Aberastury, A. (1983). Adolescência. Porto Alegre: Artes Médicas.

American Psychiatric Association (2014). Manual de Diagnóstico e Estatística de Transtornos Mentais: DSM-5. Porto Alegre, RS: Artmed

Bernal, E. P. (2019). Considerações psicanalíticas a respeito da automutilação (Dissertação de mestrado, Instituto de Psicologia da Universidade de São Paulo). Recuperado de www.teses.usp.br

Brown, R. C., \& Plener, P. L. (2017). Non-suicidal Self-Injury in Adolescence. Current Psychiatry Reports, 19(3), 20. Recuperado de http://doi. org/10.1007/s11920-017-0767-9

Cardoso, M. R., Demantova, A. G., \& Maia, G. D. C. S. (2016). Corpo e dor nas condutas escarificatórias na adolescência. Estudos de Psicanálise, 46, 115 124. Recuperado de http://pepsic.bvsalud.org/pdf/ep/n46/n46a12.pdf

Erikson, E. H. (1976). Identity: Youth and crisis. Nova York, Estados Unidos: Norton. (Trabalho original publicado em 1968) 
Fonseca, P. H. N., Silva, A. C., Araújo, L. M. C., \& Botti , N. C. L. (2018). Autolesão sem intenção suicida entre adolescentes. Arquivos Brasileiros de Psicologia, 70 (3), 246-258. Recuperado de http://pepsic.bvsalud.org/pdf/ arbp/v70n3/17.pdf

Gabriel, I. M., Costa, L. C. R., Campeiz, A. B., Salim, N. R., Silva, M. R. I., \& Carlos, D. M. (2020). Autolesão não suicida entre adolescentes: significados para profissionais da educação e da Atenção Básica à Saúde. Escola Anna Nery, 24(4), 1-9. DOI: https://doi.org/10.1590/2177-9465-EAN-2020-0050

Giusti, J. S. (2013). Automutilação: características clínicas e comparação com pacientes com transtorno obsesseivo-compulviso (Tese de doutorado, Faculdade de Medicina da Universidade de São Paulo). Recuperado de www.teses.usp.br.

Guerreiro, D. F., \& Sampaio, D. (2013). Comportamentos autolesivos em adolescentes: uma revisão da literatura com foco na investigação em língua portuguesa. Rev. Port. De Saúde Pública, 31(2), 204-213. Lisboa, Portugal. Recuperado de https://doi.org/10.1016/i.rpsp.2013.05.001

Hawton, K., Saunders, K., \& C O'connor, R. (2012). Self-harm and suicide in adolescents. The Lancet, 379, 23-29 June, p. 2373-2382. Recuperado de https://doi.org/10.1016/S0140-6736(12)60322-5

Kaplan, H. I., Sadock, B. J., \& Grebb, J. A. (1997). Compêndio de Psiquiatria: Ciências do Comportamento e Psiquiatria Clínica. Porto Alegre, RS: Artmed.

Kovács, M. J. (2008). Comportamentos autodestrutivos e o suicídio. In Kovács, M. J. (Ed.), Morte e desenvolvimento bumano (pp. 171-194). São Paulo, SP: Casa do psicólogo.

Le Breton, D. (2010). Escarificações na adolescência: uma abordagem antropológica. Horizontes Antropológicos, (16) 33, 25-40. Disponível em: http:// www.scielo.br/pdf/ha/v16n33/03.pdf

Levinsky, D. (1998). Adolescência: reflexões psicanaliticas. Porto Alegre, RS: Artes Médicas.

Lopes, L. S., \& Teixeira, L. C. (2019). Automutilações na adolescência e suas narrativas em contexto escolar. Estilos da Clínica, 24 (2), 291-303. DOI: https://doi.org/10.11606/issn.1981-1624.v24i2p291-303

Mendes, K. D. S., Silveira, R. C. C. P. \& Galvão, C. M. (2008) Revisão integrativa: método de pesquisa para a incorporação de evidências na saúde e na enfermagem. Texto Contexto Enferm, 17(4): 758-764. Recuperado de http://www. scielo.br/scielo.php?script $=$ sci_arttext\&pid $=$ S0104- 07072008000400018\&ln $\mathrm{g}=$ en\&nrm $=$ iso

Ministério da Saúde (2013) Sistema de Vigilância de Violências e Acidentes (Viva): 2009, 2010 e 2011. Brasília. Ministério da Saúde. Recuperado de http://bvs$\mathrm{ms}$.saude.gov.br/bvs/publicacoes/sistema_vigilancia_violencia_acidentes.pdf,
Ministério da Saúde (2017) Viva: Vigilância de Violências e Acidentes: 2013 e 2014 [recurso eletrônico]. Brasília. Ministério da Saúde, 2017. Recuperado de http://bvsms.saude.gov.br/bvs/publicacoes/viva vigilancia violencia acidentes 2013 2014.pdf

Moraes, D. X., Moreira, E. S., Sousa, J. M., Vale, R. R. M., Pinho, E. S., Dias, P. C. S., Caixeta, C. C. (2020). "Caneta é lâmina, minha pele o papel”: fatores de risco da automutilação em adolescentes. Revista Brasileira de Enfermagem, 73 (1), 1-9. DOI https://doi.org/10.1590/0034-7167-2020-0578

Moreira, E. S., Vale, R. R. M., Caixeta, C. C., \& Teixeira, R. A. G. (2020). Automutilação em adolescentes: revisão integrativa da literatura. Ciênc. Saúde coletiva, 25 (10), 3945-3954. DOI 10.1590/1413-812320202510.31362018

Otto, S. C., \& Santos, K. A. (2016). O Tumblr e sua relação com práticas autodestrutivas: o caráter epidêmico da autolesão. Psic. Rev. São Paulo, 25 (2), 265288. Recuperado de https://revistas.pucsp.br/psicorevista/article/view/24537

Santos, L. C. S., \& Faro, A. (2018). Aspectos conceituais da conduta autolesiva: Uma revisão teórica. Psicol. Pesqui., 12(1), 1-10. DOI: $10.24879 / 201800120010092$

Sant'Ana, I. M. (2019). Autolesão não Suicida na Adolescência e a Atuação do Psicólogo Escolar: Uma Revisão Narrativa. Revista de Psicologia da IMED, 11(1), 120-138. DOI: https://doi.org/10.18256/2175-5027.2019.v11i1.3066

Silva, A. C., \& Botti, N. C. L. (2017). Comportamento Autolesivo ao longo do ciclo vital: Revisão Integrativa da Literatura. Revista Portuguesa de Enfermagem de Saúde Mental, 18, 67-76. DOI: 10.19131/rpesm.0194

Tardivo, L. S. L. P. C. (2007). O adolescente e sofrimento emocional nos dias de hoje. São Paulo, SP: Vetor

Tardivo, L. S. L. P. C., Ferreira, L. S., Alhanat, M., Chaves, G., Rinaldi, H. R. Pinto Junior, A. A., \& Belisario, G.O. (2019a). Self-injurious behavior in preadolescents and adolescents: self-image and depression. Paripex - Indian Journal of research, 8(6), 1-5. Recuperado de https://www.worldwidejournals. com/paripex/fileview/June 2019 1559720176 0410586.pdf

Tardivo, L. S. P. C., Rosa, H. R., Ferreira, L. S., Chaves, G., \& Pinto Júnior, A. A. (2019b). Autolesão em Adolescentes, depressão e Ansiedade: um estudo compreensivo. Boletim Academia Paulista de Psicologia, 39 (97), 157-169. Recuperado de http://pepsic.bvsalud.org/pdf/bapp/v39n97/a02v39n97.pdf

Tostes, G. W., Assis, N. P., Aiello Vaisberg, T. .M J., \& Corbett, E. (2018). Dor cortante: sofrimento emocional de meninas adolescentes. Contextos Clínicos, 11(2), 257-267. DOI: $10.4013 /$ ctc.2018.112.10

Submetido em: 26-01-2021

Aceito em: 18-10-2021 


\section{Anexos}

Figura 1: Representação do fluxo de inclusão e exclusão de artigos.

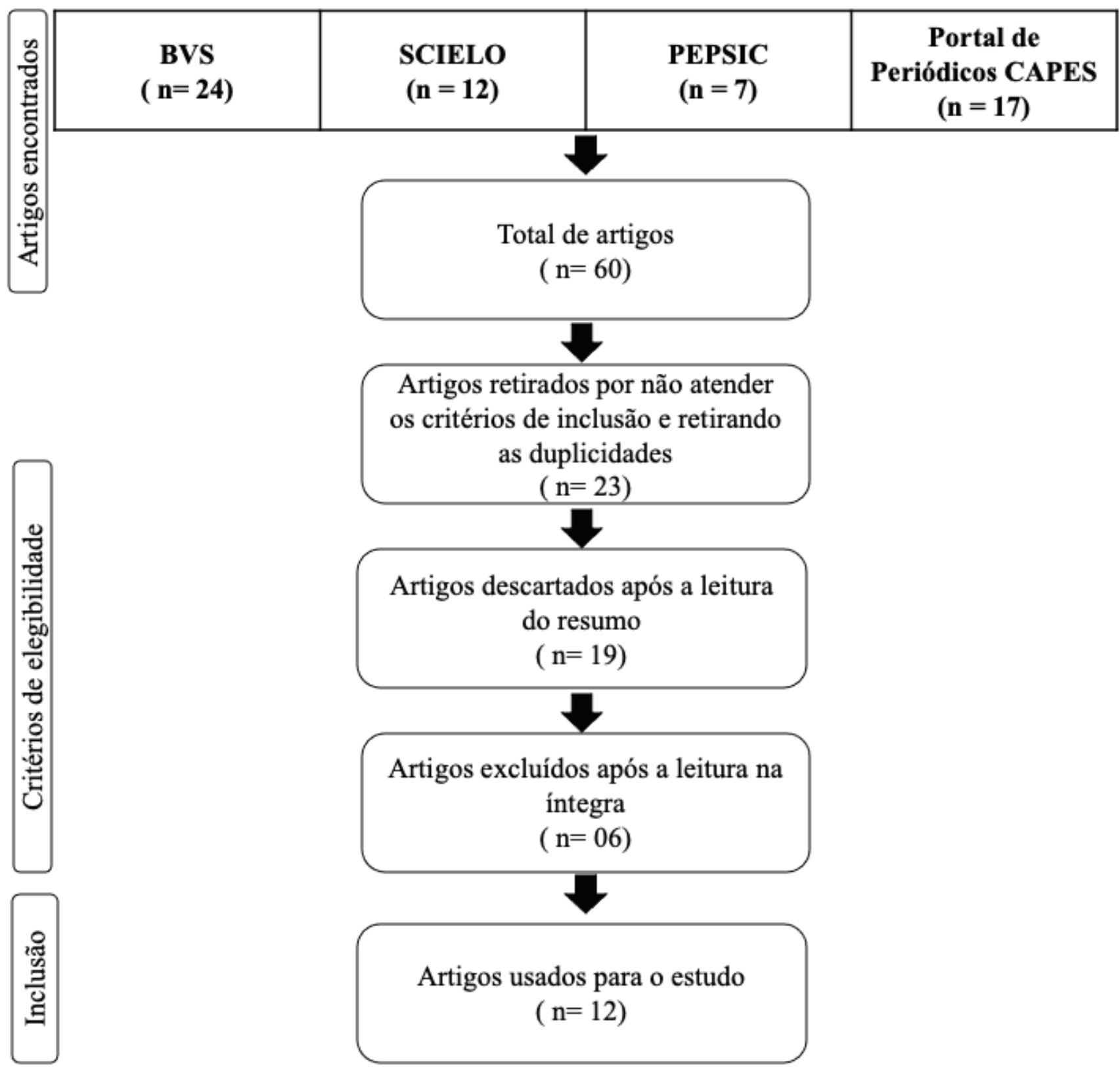


Tabela 1: Apresentação dos estudos incluídos

\begin{tabular}{|c|c|c|}
\hline Autor (ano) & Objetivos & Método \\
\hline $\begin{array}{l}\text { Otto \& Santos } \\
(2016)\end{array}$ & $\begin{array}{l}\text { Compreender se platafor- } \\
\text { mas blogging da internet } \\
\text { ('Tumblr) afetam os usuá- } \\
\text { rios quanto a práticas de } \\
\text { autolesão }\end{array}$ & $\begin{array}{l}\text { Análise do discurso } \\
\text { das publicações sobre } \\
\text { autolesão feitas em duas } \\
\text { páginas da plataforma } \\
\text { Tumblr }\end{array}$ \\
\hline $\begin{array}{l}\text { Cardoso, De- } \\
\text { mantova, \& } \\
\text { Maia (2016) }\end{array}$ & $\begin{array}{l}\text { Examinar como a autole- } \\
\text { são indica uma tentativa } \\
\text { de inscrição no corpo de } \\
\text { elementos que não con- } \\
\text { seguem ser inscritos no } \\
\text { psiquismo. }\end{array}$ & Sem dados \\
\hline $\begin{array}{l}\text { Silva \& Botti } \\
\text { (2017) }\end{array}$ & $\begin{array}{l}\text { Identificar os fatores } \\
\text { associados ao comporta- } \\
\text { mento autolesivo }\end{array}$ & $\begin{array}{l}\text { Revisão integrativa de } \\
\text { literatura realizado nas } \\
\text { bases de dados BVS, } \\
\text { IBECS, HTA, LILACS, } \\
\text { CENRTAL e MEDLINE, } \\
\text { com as palavras-chave: } \\
\text { selfharm AND fatores de } \\
\text { risco. Foram considerados } \\
\text { artigos em inglês, espa- } \\
\text { nhol e português. }\end{array}$ \\
\hline $\begin{array}{l}\text { Fonseca, Silva, } \\
\text { Araújo, \& Botti } \\
(2018)\end{array}$ & $\begin{array}{l}\text { Avaliar a frequência e a } \\
\text { gravidade da autolesão em } \\
\text { adolescentes, as variáveis } \\
\text { psicossociais e as funções } \\
\text { ou motivos que levam a } \\
\text { tal comportamento. }\end{array}$ & $\begin{array}{l}\text { Pesquisa realizada com } \\
517 \text { adolescentes, entre } \\
10 \text { e } 14 \text { anos, utilizando } \\
\text { como instrumento a Es- } \\
\text { cala de Comportamento } \\
\text { de Autolesão (ECA) }\end{array}$ \\
\hline
\end{tabular}

Resultados

Plataforma Tumblr como lugar de troca e expressão, considerado livre de julgamento para a prática da autolesão e do sofrimento que esse comportamento acarreta.

A dor causada pelos cortes constitui um recurso defensivo arcaico na tentativa do ego se apropriar desse corpo.

Autolesão com um ato não fatal (sem intenção suicida consciente), embora grave e um fator de risco para o suicídio e pode estar relacionada a transtornos limítrofes de personalidade, depressão, ansiedade, abusos de substâncias e transtornos alimentares. É mais frequente na adolescência e pode ser agravada quando o indivíduo está exposto à violência, por exemplo bullying.

Mais frequente no público feminino $(69,39 \%)$, $9,48 \%$ relataram ter praticado a autolesão cinco vezes ou mais vezes no ano. 61,22\% dos adolescentes não associaram a autolesão com intenção suicida, tampouco estavam sob efeito de drogas quando a praticaram $(97,69 \%)$ e $69,39 \%$ afirmaram sentir dor ao realizar os cortes, que foram compreendidos pelos adolescentes como forma de aliviar a sensação de vazio ou indiferença e cessar sentimentos ou sensações ruins.

Santos \& Faro Reunir os principais (2018) achados teóricos sobre aspectos conceituais, epidemiológicos, clínicos e terapêuticos do comportamento autolesivo.
Revisão narrativa da literatura de trabalhos publicados nas bases de dados Web of Science, SciELO, Pepsic e Google Scholar, utilizando as palavras-chave autoinjúria, autodano, autolesão, cutting, caving, parassuicídio, dano autoinfligido, conduta autolesiva e comportamento autolesivo.
Os estudos demonstraram que o comportamento autolesivo tem maior prevalência na adolescência e podem estar relacionados aos contextos estressores ou traumáticos na infância, influência prejudicial do meio social, do ambiente e dos pares, concluindo que esse é um comportamento multicausal. OS cortes foram compreendidos como forma de regular/ minimizar afetos negativos e comunicar ou sinalizar o sofrimento. 


\begin{tabular}{ll}
\hline Autor (ano) & Objetivos \\
\hline Tostes, Assis, & Estudar a autolesão em \\
Aiello Vais- & meninas adolescentes, \\
berg \& Corbett & $\begin{array}{l}\text { compreendendo-a como } \\
\text { (2018) }\end{array}$ \\
& $\begin{array}{l}\text { expressão de sofrimento } \\
\text { emocional. }\end{array}$
\end{tabular}

Tardivo, Rosa, Compreender os aspectos Ferreira, Chaves, psicológicos em ado\& Pinto Júnior (2019b)

Lopes \& Teixeira (2019)

Sant'Ana (2019)

Gabriel, Costa, Campeiz, Salim, Silva, \& Carlos (2020)
Analisar a autolesão em adolescentes e como essas narrativas surgem na escola. visão de si, do mundo, as relações, angústias, emoções, e identificar sinais de depressão e ansiedade. autolesiva, considerando a

\author{
Resultados \\ Os adolescentes relataram viver em ambiente \\ hostil, marcado por experiências de não se senti- \\ rem amados e considerados. Foram encontrados \\ dois campos afetivos-emocionais: "desprovidas \\ de afeto" (crença - ou fantasia - de que o outro \\ lhe deve afeto e atenção) e "crime e castigo" \\ (crença - ou fantasia - de que as pessoas que \\ se comportam mal devem ser castigadas, o que \\ inclui a privação de afeto).
}

Histórias marcadas por sentimentos de desproteção, tristeza e solidão, associado por falhas, privação, negligência, ausência de um dos pais e graves problemas na estrutura familiar, que dificultam a comunicação e vínculo. Os cortes foram apresentados como uma forma de buscar "alívio", de comunicar o sofrimento e pedido de proteção, ajuda, de ser cuidado e compreendido. Os instrumentos demonstraram presença de sintomas depressivos e sinais de ansiedade.

Escuta clínica, utilizando-se da associação livre, realizada com uma adolescente no ambiente escolar.

Autolesão como tentativa de lidar com conflitos, os quais podem ter diversos motivos (relação conflituosa dos pais, término do namoro, não estar se saindo bem na escola e prazer em sentir a dor). O trabalho apresenta a escuta como forma de verbalizar as angústias em um movimento criativo.

Revisão narrativa utilizando as bases de dados: SciELO, PePSIC, Catálogo de Teses e Dissertações da Comissão de Aperfeiçoamento de Pessoal do Nível Superior (CAPES) e Google, com os descritores: autolesão, automutilação, adolescência e psicologia escolar.

Grupo focal com profissionais da saúde de uma Unidade de Saúde da Família (USF) e profissionais da educação de uma escola estadual de ensino fundamental e médio, todos com contato com adolescentes que se autolesionam.
O trabalho da Psicologia Escolar com alunos que apresentem o comportamento autolesivo envolve os alunos, famílias e educadores. No entanto, a atuação desses profissionais é marcada por desafios, tanto pela ausência do psicólogo no sistema educacional público, como outros problemas do âmbito educacional (evasão escolar, precarização do trabalho docente, fracasso escolar).

Os participantes identificaram como motivos da autolesão: mudanças e descobertas que o adolescente vivencia, aspectos hormonais, cortes como uma forma de "chamar a atenção" e efeito do contágio agravado pela tecnologia, dinâmica familiar e a falta de diálogo com o adolescente. Quanto às formas de intervenção, apresentaram as notificações e encaminhamentos para profissionais da saúde, assim como a escuta e acolhimento tanto pelos profissionais da saúde quanto pelos professores. 
REVISÃO INTEGRATIVA DA LITERATURA

\begin{tabular}{|c|c|c|c|}
\hline Autor (ano) & Objetivos & Método & Resultados \\
\hline $\begin{array}{l}\text { Moraes, Morei- } \\
\text { ra, Sousa, Vale, } \\
\text { Pinho, Dias, \& } \\
\text { Caixeta }(2020)\end{array}$ & $\begin{array}{l}\text { Descrever os fatores de } \\
\text { risco que influenciam } \\
\text { o comportamento da } \\
\text { automutilação em adoles- } \\
\text { centes. }\end{array}$ & $\begin{array}{l}\text { Grupo focal, utilizando } \\
\text { perguntas norteadoras } \\
\text { sobre autolesão, com sete } \\
\text { adolescentes que apresen- } \\
\text { tavam o comportamento, } \\
\text { atendidos em um CAPSi. } \\
\text { A seleção e análise dos } \\
\text { participantes foi por } \\
\text { meio dos prontuários do } \\
\text { serviço. }\end{array}$ & $\begin{array}{l}\text { Cinco participantes apresentaram algum tipo de } \\
\text { transtorno de humor (transtorno afetivo bipolar } \\
\text { ou depressão) e duas transtorno de ansieda- } \\
\text { de. Fatores de risco encontrados: dificuldades } \\
\text { nas relações familiares, contato com pessoas } \\
\text { que se autolesionam (amigos ou mídias so- } \\
\text { ciais), bullying, violência sexual e características } \\
\text { individuais (baixa autoestima, dificuldade de se } \\
\text { expressar, isolamento social, orientação sexual, } \\
\text { tristeza e ideação suicida). }\end{array}$ \\
\hline $\begin{array}{l}\text { Moreira, Vale, } \\
\text { Caixeta, \& Tei- } \\
\text { xeira }(2020)\end{array}$ & $\begin{array}{l}\text { Síntese das produções } \\
\text { científicas sobre automuti- } \\
\text { lação em adolescentes. }\end{array}$ & $\begin{array}{l}\text { Revisão integrativa da } \\
\text { literatura realizada nas } \\
\text { bases de dados Scielo } \\
\text { e Portal de Periódicos } \\
\text { CAPES no período de } \\
\text { janeiro de } 2012 \text { a junho } \\
\text { 2017, nos idiomas portu- } \\
\text { guês, inglês e espanhol, a } \\
\text { partir dos descritores: au- } \\
\text { tomutilação e adolescen- } \\
\text { tes. Foram considerados } \\
\text { adolescentes indivíduos } \\
\text { entre } 10 \text { e } 21 \text { anos. }\end{array}$ & $\begin{array}{l}\text { Identificada escassez de estudos sobre a autole- } \\
\text { são no sexo masculino e falta de consenso entre } \\
\text { os termos usados (Non-suicidal self-injury foi o } \\
\text { mais utilizado). A autolesão foi compreendida } \\
\text { como forma de sentir algo, regular emoções ne- } \\
\text { gativas, associada ao quadro de depressão, fobia } \\
\text { social, Transtorno de Estresse Pós-traumático, } \\
\text { de Ansiedade, comportamentos suicida, transtor- } \\
\text { nos alimentares e de personalidade borderline. } \\
\text { Os fatores de risco relacionados a autolesão } \\
\text { foram: violência, bullying, excesso de álcool e } \\
\text { drogas, dificuldade no relacionamento familiar } \\
\text { (principalmente com a figura materna), conhecer } \\
\text { outras pessoas com o comportamento, impulsivi- } \\
\text { dade, baixa autoestima e dificuldade de expressar } \\
\text { emoções. A pesquisa ressalta a carência de estu- } \\
\text { dos que testem a eficácia terapêutica e estratégias } \\
\text { de prevenção. }\end{array}$ \\
\hline
\end{tabular}

